\title{
Evaluation of Adverse Effects of Topical Glaucoma Medications on Trabeculectomy Outcomes Using the Glaucoma Medications Intensity Index
}

\author{
Jasper Ka Wai Wong · Tsz Kin Leung · Jimmy Shiu-ming Lai • \\ Jonathan Cheuk-hung Chan (D)
}

Received: October 1, 2021 / Accepted: December 7, 2021 / Published online: December 21, 2021

(c) The Author(s) 2021

\begin{abstract}
Introduction: Trabeculectomy is commonly performed for glaucoma when medications are unable to control disease progression or have intolerable adverse effects. Previous studies have suggested that a higher number of and/or longer treatment duration with preoperative topical glaucoma medications are associated with a higher risk of trabeculectomy failure, but most of these studies lack quantification of exposure. The aim of this study was to investigate the relationship between preoperative exposure to topical glaucoma medications and trabeculectomy outcome, using a new method for quantifying accumulated exposure.

Methods: Consecutive patients with primary open-angle glaucoma (POAG) or normal-tension glaucoma (NTG) who underwent primary trabeculectomy between 2013 and 2017 were retrospectively reviewed. The Glaucoma Medications Intensity Index (GMII) was calculated for each eye by multiplying the number of drops per week by duration of use (in years). The
\end{abstract}

J. K. W. Wong · J. S. Lai · J. C. Chan (ه)

Department of Ophthalmology, Li Ka Shing Faculty of Medicine, University of Hong Kong, Room 301, Block B, Cyberport 4, Hong Kong, Hong Kong e-mail: jonochan@hku.hk; jonnochan@yahoo.com

T. K. Leung

Li Ka Shing Faculty of Medicine, University of Hong Kong, Hong Kong, Hong Kong relationship between the GMII and postoperative outcome in terms of 1- and 2-year success rates and survival time was analyzed.

Results: A total of 55 eyes from 40 subjects were analyzed, all with follow-up $>6$ months (mean $2.72 \pm 1.46$ years). The GMII for eyes with successful $(n=41)$ and failed $(n=14)$ outcome at last visit was $111.71 \pm 78.59$ and $167.41 \pm 85.04$, respectively, and significantly higher in failed eyes $(P=0.03)$. Univariate regression analysis of age, gender, cup-disc ratio, previous phacoemulsification, diabetes, hypertension, dyslipidemia, preoperative number of glaucoma medications/treatment duration/intraocular pressure (IOP), and GMII showed age and GMII to be possible predictors of treatment failure. On subsequent multivariate analysis, only GMII was correlated with failure (odds ratio 1.021, 95\% confidence interval 1.00-1.05; $P=0.05$ ). When GMII $\geq 80$, the postoperative survival time was shorter $(P=0.02)$, the 1 -year IOP, number of glaucoma medications, and number of needlings performed were higher $(P=0.03, P<0.01, P<$ 0.03 , respectively), and reduction in glaucoma medication was less $(P=0.02)$.

Conclusion: The GMII can be used to predict eyes at higher risk for trabeculectomy that may benefit from additional perioperative intervention or treatment. It can also help the surgeon time the surgery before the GMII becomes too high, thereby optimizing the patient's postoperative outcome. 
Keywords: Filtration surgery outcome; Glaucoma medications; Medication exposure; Trabeculectomy outcome; Treatment intensity

\section{Key Summary Points}

Why carry out this study?

The success of many current glaucoma surgeries (including trabeculectomy) are dependent on the preoperative conjunctival status, which is adversely affected by exposure to topical glaucoma medications.

Most eyes undergoing glaucoma surgery have had long-term exposure to topical glaucoma medications, but the effect of this exposure has not yet been accurately quantified.

Our aim was to quantify the threshold exposure to preoperative topical glaucoma medications that is associated with increasingly poor postoperative outcome, to help predict high-risk cases.

\section{What was learned from the study?}

The use of the Glaucoma Medication Intensity Index (GMII) allows a more accurate assessment of preoperative exposure to topical glaucoma medications.

A preoperative GMII $\geq 80$ was associated lwith lower trabeculotomy success in terms of postoperative survival time, intraocular pressure, reduction in medications, and subsequent requirement for needling procedures.

Use of the preoperative GMII to predict high-risk trabeculectomy cases can allow the surgeon to plan accordingly and optimize the patient's postoperative outcome.

\section{INTRODUCTION}

Glaucoma, together with macular degeneration, are two of the leading causes of irreversible blindness worldwide [1]. In 2010, 2.1 million and 4.2 million people were reported to be blind or visually impaired due to glaucoma, respectively, corresponding to $6.6 \%$ cases of blindness and $2.2 \%$ cases of moderate to severe visual impairment globally [1]. In 2013, the total number of people with glaucoma was estimated at 64.3 million [2]. The goal of glaucoma treatment is to prevent progression, and intraocular pressure (IOP) is the most significant modifiable risk factor $[3,4]$. The aim of current glaucoma management strategies are to reduce IOP [5] to a level where disease progression or development can be delayed or even prevented $[6,7]$.

There are several options for reducing IOP in glaucoma. Due to the risk of potentially sightthreatening complications [8] and the unpredictable long-term outcome of glaucoma surgery [9], most newly diagnosed patients are treated initially with topical medications and/or laser trabeculoplasty, followed by surgery. Trabeculectomy and glaucoma drainage implants are usually performed when the IOP remains uncontrolled by non-surgical measures [10]. Therefore, most glaucoma patients undergoing trabeculectomy have a long history of using topical glaucoma medications preoperatively.

It has been reported that the conjunctiva is adversely affected not just by topical glaucoma medications, but also by the accompanying preservatives [11-15]. These changes include increased numbers of macrophages, lymphocytes, and fibroblasts within the conjunctiva, suggesting that subclinical chronic inflammation is associated with the use of topical glaucoma eye drops [12, 15]. This inflammatory effect may explain the poorer outcome from trabeculectomy in eyes treated preoperatively with glaucoma eye drops compared to those that underwent early trabeculectomy $[12,16]$. However, it is unknown whether this adverse effect on trabeculectomy by glaucoma eye drops occurs with any level of exposure or if it is dose dependent. 
In this study, we retrospectively reviewed consecutive patients from 2013 to 2017 who underwent primary trabeculectomy at two hospitals (but same department) in Hong Kong, to determine whether trabeculectomy outcome is dependent on the total accumulated exposure or dose of preoperative topical glaucoma medications.

\section{METHODS}

\section{Study Design}

Ethics approval for this study was obtained from the Institutional Review Board of the University of Hong Kong/Hospital Authority Hong Kong West cluster (reference number: UW 18-487). The study was conducted in accordance with the Declaration of Helsinki of 1964 and its later amendments.

All cases of trabeculectomy (International Classification of Diseases, Ninth Revision [ICD9] code: 12.64 ) performed from 2013 to 2017 by the Department of Ophthalmology of Hong Kong West cluster, at Grantham Hospital or Queen Mary Hospital, were identified using the Clinical Data Analysis and Reporting System (CDARS) of the Hospital Authority of Hong Kong. The medical records of each eye were reviewed for eligibility depending on their fulfilment of the inclusion and exclusion criteria using the centralized, electronic patient record (EPR) system of the Hospital Authority.

The inclusion criteria were: (1) primary open-angle glaucoma (POAG), normal tension glaucoma (NTG), or ocular hypertension (OHT); (2) primary trabeculectomy with mitomycin-C (MMC), with or without combined phacoemulsification; (3) age $\geq 18$ years at the time of surgery; and (4) follow-up of at least 3 months after surgery.

The exclusion criteria were: (1) prior surgical procedures (including previous trabeculectomy), apart from uncomplicated clear cornea phacoemulsification; (2) prior laser cyclophotocoagulation, iridoplasty, or iridotomy; (3) prior laser trabeculoplasty within 4 weeks of trabeculectomy (NOT excluded if performed $>$ 4 weeks before trabeculectomy); (4) prior ocular cryotherapy (including cyclocryotherapy and retinal cryopexy); and (5) missing clinical data for analysis due to incomplete medical record (including patients initiated on glaucoma treatment elsewhere).

As primary trabeculectomy without intraoperative MMC is very rarely performed in our locality, it is unlikely any suitable subjects would be excluded because of this inclusion criteria.

All trabeculectomy procedures were performed by one of six experienced (post-fellowship) glaucoma specialists from the same department, using a standardized fornix-based approach and applying sterile cellulose sponges soaked with $0.4 \mathrm{mg} / \mathrm{mL}$ MMC for 3 min directly over the scleral bed and under the Tenon's capsule, prior to making the scleral flap. None of the eyes had received any pre-medication (steroid or non-steroidal anti-inflammatory drug) before the surgery. Postoperatively, all subjects were followed in the glaucoma clinic, and the study eye received routine postoperative treatment with topical antibiotic and steroid, or with a combination antibiotic/steroid formulation (levofloxacin $0.5 \%+$ prednisolone acetate $1 \%$, or combined dexamethasone $0.1 \%+$ neomycin $0.5 \%$ ), four to eight times per day for 4-12 weeks, according to clinical indications.

Each study eye was recorded separately for subjects with both eyes eligible. The following information was collected for each subject or study eye:

- Subject:

- gender

- age (at the time of surgery)

- ethnicity

- Study eye:

- laterality

- cup-disc ratio (CDR)

- type of glaucoma (POAG, NTG or OHT)

- surgical details 
- pre- and postoperative visual acuity (VA), IOP, and glaucoma medications

- any co-existing ocular pathology

- any postoperative ocular adverse event, whether related or not to the trabeculectomy

\section{Preoperative Topical Glaucoma Medication Exposure Measurement}

We adopted and modified the treatment intensity index from Saade et al. [17], which we renamed as the Glaucoma Medication Intensity Index (GMII) to differentiate it from its simpler predecessor. In the study by Saade, the intensity index (the total number of all eye drops applied per week, multiplied by treatment duration in years) was used to represent the total treatment or exposure load to the eye up to the study time point. However, it was uncertain how the calculation was done when the number of drops varied over time; i.e., whether the "number of drops per week" was an average or median for the entire treatment duration, whether proportional weighting based on actual usage duration was done, or whether the calculation was just based on the treatment regimen at recruitment (even though many patients initially started on one medication may have been using more than one by the time of recruitment).

In this study, we clarified the preoperative exposure load by subdividing the total treatment duration into different time intervals whenever the number of drops used varied. The number of drops used per week during a particular interval would then be multiplied by the duration of that specific treatment (in years or fraction of a year) to obtain a sub-index. The final index is the sum of all sub-indices:

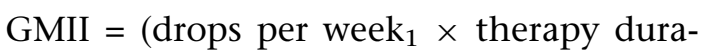
tion in years 1$)+\left(\right.$ drops per week $_{2} \times$ therapy duration in years $\left.{ }_{2}\right)+\left(\right.$ drops per week $_{3}$ $x$ therapy duration in years $\left.{ }_{3}\right)+\ldots \ldots$.

For example, if the right eye of a patient has been treated with glaucoma eye drops for 10 years, with 7 drops per week during the first 4 years, followed by 21 drops per week during the last 6 years, then the GMII for that eye is ( 7 $\times 4)+(21 \times 6)=154$. The GMII was calculated for all eligible eyes in this study. In the GMII calculation, fixed-combination eye drops were counted as one drop for each application, irrespective of the number of component medications within that drop.

\section{Definition of Medication Exposure, Medication Duration, and Medication Requirement}

For the definition of "medication exposure", we counted each fixed-combination of glaucoma eye drops used preoperatively as a single medication, similar to the study by Saade et al. [17] and as used in our GMII calculation. "Medication duration" is simply the duration of preoperative glaucoma eye drop use in the study eye. While both medication exposure and duration are related to GMII, they are intentionally included in our analysis as "controls" to compare their predictive performance (for trabeculectomy failure) with GMII. To avoid confusion, we used the term "medication required" or "medication requirement" for defining the actual number of medications used, so that each fixed-combination of eye drops is counted as two medications.

This delineation of medication exposure and duration (determined preoperatively) from medication requirement (determined both preoperatively and postoperatively) can better reflect the degree of preoperative exposure than counting each component separately for a fixed-combination of eye drops, as one of the major advantage of these formulations is reduction of the total number of drops used, even for preservative-free eye drops (for example, a prostaglandin analog and a beta-blocker is 3 drops/day in total; a combination formulation reduces this to 1 drop/day).

The use of "medication requirement" in our study is mainly for analyzing success criteria, comparing the preoperative with the postoperative requirement. Obviously, the actual number of glaucoma medications required for IOP control would be needed for such analysis since an eye requiring a prostaglandin analog/betablocker fixed-combination drop to achieve "success" status would be less successful than 
another eye requiring monotherapy with a prostaglandin analog drop only.

\section{Outcome Measurement}

Failure criteria were defined as:

- IOP $>21 \mathrm{mmHg}$ or $<20 \%$ reduction on two consecutive visits at least 3 months after initial trabeculectomy, with or without use of glaucoma medications

- Requirement for an additional procedure to reduce IOP (including tube-shunt, repeat trabeculectomy, cyclophotocoagulation), except for:

- lysis, cutting, or removal of scleral flap sutures

- subconjunctival 5-fluorouracil or MMC injections

- bleb needling

- loss of light perception (because of the trabeculectomy, or a complication of this procedure)

Eyes fulfilling any of the above failure criteria postoperatively were recorded at that time point to have failed, along with the survival duration (time from initial trabeculectomy). Eyes which have not fulfilled any of the above failure criteria at the relevant study time points were deemed to be successful.

- Primary outcome:

- Success rate at the 1-year and 2-year time points

- Secondary outcome:

- Survival time

- duration between the initial trabeculectomy to the occurrence of any one of the above failure criteria is recorded as the survival time

- Other individual indicators of postoperative success:

- IOP

- bleb-needling procedure performed

- glaucoma medication requirement

- Effect of different types of preservative exposure on postoperative success

\section{Statistical Analysis}

Two-sample equal and unequal variance $t$-tests were used to compare the mean of continuous data, depending on whether the two groups of data have equality of variance from the $F$-test. Univariate and multivariate regression analyses were used to test for the association between various continuous and categorical variables with trabeculectomy outcome. A $P$ value $\leq 0.05$ was considered to be statistically significant. Survival analysis and log-rank test were also performed to compare the survival distributions of eyes with GMII above and below an estimated cutoff value.

\section{RESULTS}

Of the 126 trabeculectomies performed from 2013 to 2017,71 were excluded from the study, mostly due to the diagnosis being angle closure glaucoma or uveitic glaucoma, the eye had had previous glaucoma procedures apart from laser trabeculoplasty, or the procedure was incorrectly coded as trabeculectomy (occasionally occurs with deep sclerectomy and trabectome). This left a total of 55 eyes of 40 subjects (all were Chinese) with a mean overall postoperative follow-up duration ( \pm standard deviation $[\mathrm{SD}]$ ) of $2.72 \pm 1.46$ (range 0.60-6.88) years. There were 27 right and 28 left eyes, with 44 having POAG and 11 with NTG (there was no OHT). The mean preoperative IOP and medications 
required overall was $22.89 \pm 7.13 \mathrm{mmHg}$ and $3.51 \pm 0.77$, respectively. The other preoperative characteristics of the subjects and study eyes, overall and according to outcome at final visit, are summarized in Table 1 . Most eyes (50/ 55) had baseline Snellen VA better than 20/200; there were two eyes with 20/200, and one each with 20/1200, finger counting and hand movement only. Overall, 14 eyes had failed by the last visit (all had follow-up duration > 6 months). Five (2.5\%) and ten (25.0\%) subjects, or six (10.9\%) and $19(34.5 \%)$ eyes, that were lost to follow-up before the 1-year and 2 -year visits, respectively. By the 1-year and 2-year time points, nine (of 49) and 12 (of 36) eyes had reached failure criteria, respectively. As there was only one case of concurrent phacoemulsification, with the eye reaching failure criteria after 33 weeks and with a GMII of 120 , this preoperative variable was not used in subsequent analyses. None of the subjects were documented in the medical record as being non-compliant with their prescribed glaucoma treatment for any significant period of time.
The preoperative mean age, GMII, treatment duration, medication exposure, medication requirement, CDR, and IOP were assessed for association with surgical failure at 1 and 2 years postoperatively and at the last follow-up visits, as summarized in Table 2. The definitions for medication exposure and requirement were as stated in the Methods section, and the preoperative value is the number that was used in the study eye just prior to the trabeculectomy procedure. Comparison of these values between the failed and successful eyes showed that only age and GMII were significantly higher in the failed eyes at the 1-year follow-up and the last visit. As expected, the number of failures increased over time, with nine failures at the 1-year follow-up, 11 at the 2-year follow-up, and 14 by the last visit, with failed cases having significantly longer follow-up duration than the successful ones. The preliminary univariate logistic regression analysis of preoperative variables on trabeculectomy failure (age, gender, CDR, previous phacoemulsification, diabetes (DM), systemic hypertension (HT), dyslipidemia,

Table 1 Preoperative characteristics of subjects and study eyes according to their outcome at last visit

\begin{tabular}{llll}
\hline Characteristics & Success & Failure & Overall \\
\hline Number of eyes & 41 & 14 & 55 \\
Age (years) & $64.68 \pm 10.08$ & $72.29 \pm 12.05$ & $66.62 \pm 11.02$ \\
Female:male & $9: 32$ & $6: 8$ & $15: 40$ \\
Right:left eyes & $21: 20$ & $6: 8$ & $27: 28$ \\
POAG:NTG & $33: 8$ & $13: 1$ & $46: 9$ \\
CDR & $0.84 \pm 0.10$ & $0.80 \pm 0.14$ & $0.83 \pm 0.11$ \\
Intraocular pressure (mmHg) & $22.32 \pm 6.04$ & $24.57 \pm 9.72$ & $22.89 \pm 7.13$ \\
Medication requirement & $3.49 \pm 0.68$ & $3.57 \pm 1.02$ & $3.51 \pm 0.77$ \\
Previous phacoemulsification & 11 & 8 & 19 \\
Concurrent phacoemulsification & 0 & 1 & 1 \\
Diabetes & 11 & 1 & 12 \\
Systemic hypertension & 24 & 7 & 31 \\
Dyslipidemia & 15 & 4 & 19 \\
\hline
\end{tabular}

Values in table are presented as the mean \pm standard deviation (SD) or as the frequency (number of eyes or patients) $C D R$ Cup-disc ratio, $N T G$ normal tension glaucoma, $P O A G$ primary open-angle glaucoma 


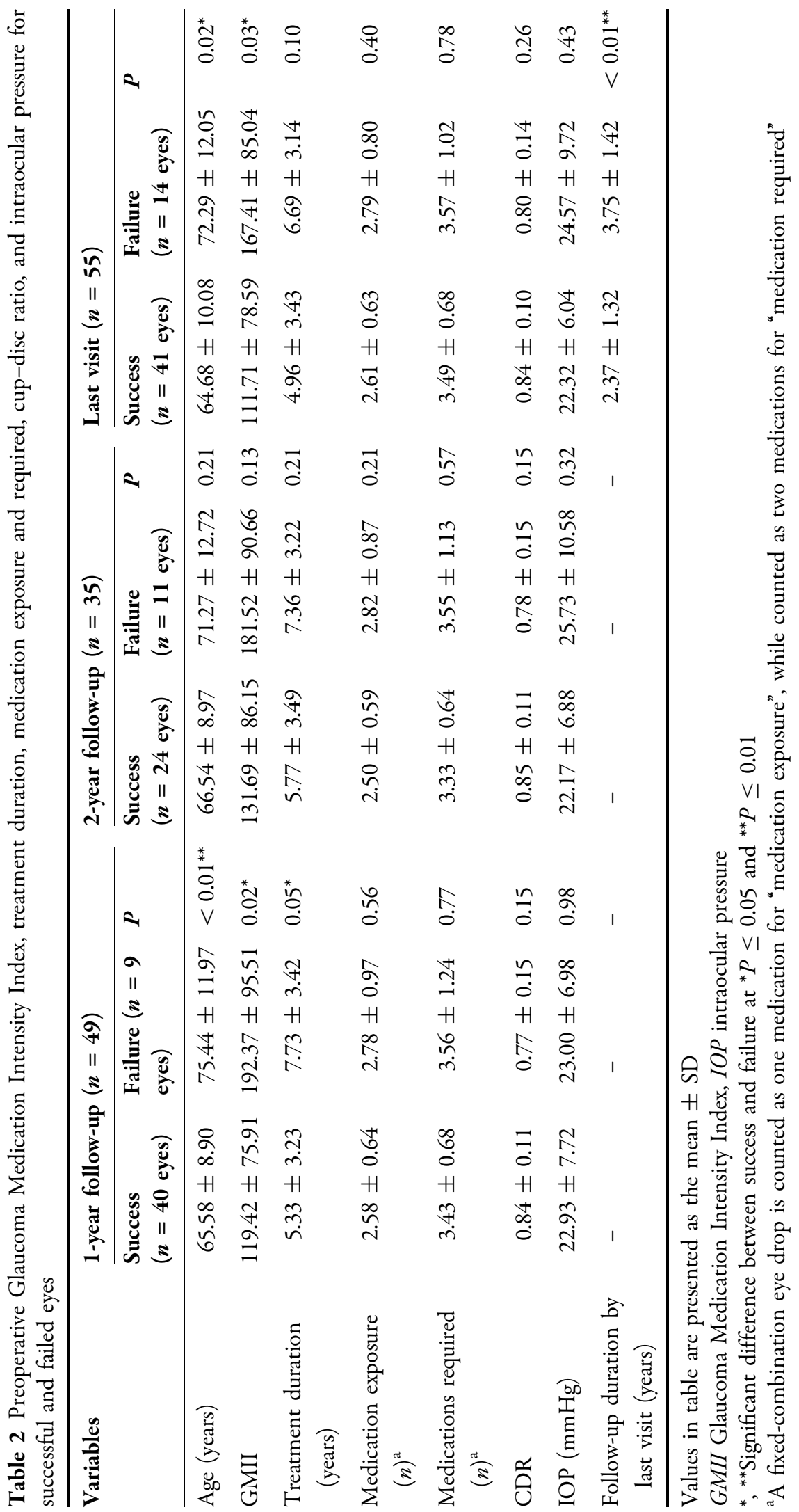


medication exposure, medication requirement, treatment duration, IOP, GMII) revealed that for eyes followed for $\geq 1$ year, three were possibly correlated $(P \leq 0.15)$ and, therefore, candidates for further multivariate analysis [18]: age $(P=0.04)$, previous phacoemulsification $(P=0.07)$ and GMII $(P=0.08)$. Subsequent multivariate analysis using a backward stepwise regression model with the lowest Akaike information criterion (AIC) of 58.26 and age, CDR, DM, (HT), treatment duration, and GMII as covariables found that only GMII was a significant predictor of failure (odds ratio [OR] 1.021, 95\% confidence interval [CI] 1.002-1.046; $P=0.05$ ), while age no longer was (Table 3 ). Using age, history of previous phacoemulsification, and GMII (all $P<0.15$ on univariate analysis) as covariables in another multivariate model with a higher AIC of 59.38 (hence less predictive performance) showed that none of these variables were significantly correlated with failure (all $P>0.05$ ).

Although the mean GMII was significantly higher in failed eyes, there was a large overlap of
GMII values between the failed and successful group, as shown in Fig. 1. After plotting the GMII values of all study eyes in the same broken-line graph, we observed that most failed eyes had GMII $\geq 80$, which is close to the value (74.32) corresponding to the point with the highest Youden's Index on the receiver operating characteristic curve for predicting failure with GMII. With further testing using both GMII $=75$ and GMII $=80$ as cutoff values in logistic regression analyses for a "high" GMII (defined as either GMII $\geq 75$ or $\geq 80$ ) as possible predictors for failure at the 1-year follow-up, we noted that GMII $\geq 80$ (OR 9.75, 95\% CI $1.66-187 ; P=0.04)$ was a better predictor of failure than GMII $\geq 75$ (OR 7.68, 95\% CI $1.29-147 ; P=0.06)$. We therefore chose the cutoff value of 80 to categorize GMII as being low (GMII $<80$ ) or high $(\geq 80)$. Using this GMII cutoff to divide our study eyes into two groups, those with a low and high preoperative GMII, respectively, at the last follow-up visit ( $n=55$, all with follow-up $>6$ months), the success rate of the low GMII group (mean follow-up

Table 3 Logistic regression analysis of preoperative factors predicting failure of trabeculectomy at the 1-year follow-up

\begin{tabular}{|c|c|c|c|c|c|c|c|c|}
\hline \multirow[t]{2}{*}{ Variables } & \multicolumn{4}{|c|}{ Univariate analysis } & \multicolumn{4}{|c|}{ Multivariate analysis } \\
\hline & $\overline{\text { OR }}$ & $95 \%$ LB & $95 \%$ UB & $P$ value & $\overline{\mathrm{OR}}$ & 95\% LB & $95 \%$ UB & $P$ value \\
\hline Age (years) & 1.07 & 1.01 & 1.15 & $0.04^{*}$ & 1.07 & 1.00 & 1.17 & 0.08 \\
\hline Gender (male) & 0.46 & 0.12 & 1.73 & 0.24 & & & & \\
\hline CDR & 0.97 & 0.93 & 1.03 & 0.30 & 0.94 & 0.87 & 1.00 & 0.06 \\
\hline Previous phacoemulsification & 3.33 & 0.93 & 12.66 & 0.07 & & & & \\
\hline Diabetes & 0.22 & 0.01 & 1.38 & 0.17 & 0.16 & 0.01 & 1.61 & 0.18 \\
\hline Systemic hypertension & 0.67 & 0.19 & 2.35 & 0.52 & 0.25 & 0.03 & 1.32 & 0.13 \\
\hline Dyslipidemia & 0.87 & 0.20 & 3.28 & 0.85 & & & & \\
\hline Medication exposure & 1.68 & 0.68 & 4.51 & 0.28 & & & & \\
\hline Medication requirement & 1.33 & 0.60 & 3.12 & 0.49 & & & & \\
\hline Treatment duration (years) & 1.12 & 0.93 & 1.36 & 0.23 & 0.68 & 0.39 & 1.08 & 0.13 \\
\hline IOP $(\mathrm{mmHg})$ & 1.04 & 0.96 & 1.13 & 0.34 & & & & \\
\hline GMII & 1.01 & 1.00 & 1.02 & 0.08 & 1.02 & 1.00 & 1.05 & $0.05^{*}$ \\
\hline
\end{tabular}

Multivariate model based on lowest Akaike information criterion (AIC) with backward stepwise selection $L B$ Lower bound, $O R$ odds ratio, $U B$ upper bound 


\section{Glaucoma Medication Intensity Index (GMII) Distribution of All Subjects}

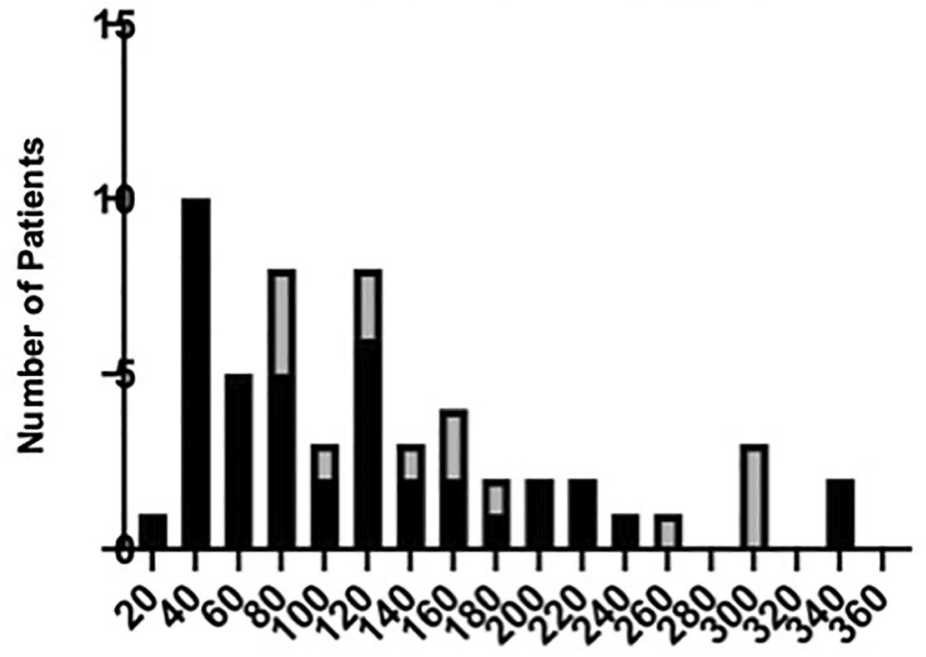

$\square$ Failure

Success

\section{Glaucoma Medication Intensity Index (GMII)}

Fig. 1 Distribution of the GMII among all subjects, showing overlap of failed (gray portion of bar) and successful (black portion of bar) cases

$2.43 \pm 1.75$ years) was $95 \%$, and significantly higher than the high GMII group's (mean follow-up $2.89 \pm 1.40$ years) success rate of $63 \%$ $(P<0.01)$, while there was no significant difference between the duration of follow-up of the two group $(P=0.27)$. At the 1-year time point, the success rate was $94 \%$ in the low GMII group $(n=16)$ and $76 \%$ in the high GMII group $(n=33)$, while at the 2-year time point, the success rate was $90 \%$ in the low GMII group $(n=10)$ and $56 \%$ in the high GMII group $(n=25)$, although the differences in success rates were not statistically significant in either case (1-year $P=0.13,2$-year $P=0.06$ ). We also performed survival analysis for both groups. The respective Kaplan-Meier survival curves in Fig. 2 showed a significantly lower survival (higher failure) rate for eyes with a high GMII (log-rank, $P=0.02$ ). When we repeated univariate logistic regression analysis using GMII as a categorical variable (low or high, instead of a continuous variable), apart from a high GMII (as noted above), increasing age (OR 1.07, 95\% CI 1.01-1.15; $P=0.04$ ) was also associated with higher odds of failure at the 1-year follow-up, as shown in Table 4.

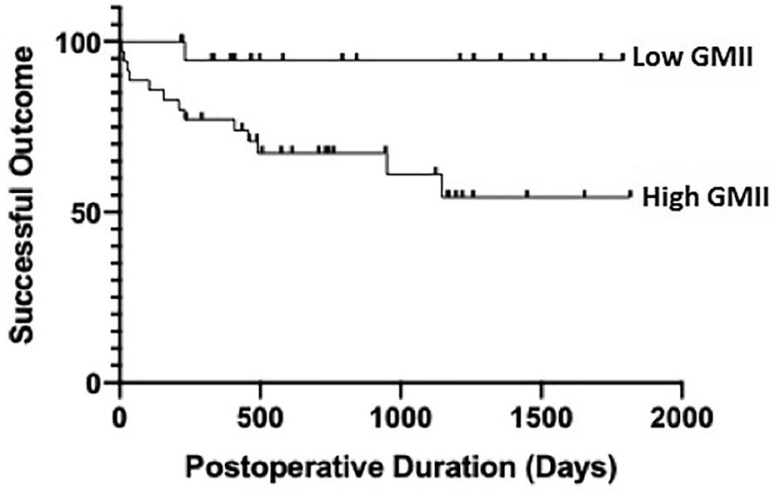

Fig. 2 Kaplan-Meier survival curves of eyes with low $(<80)$ and high $(\geq 80)$ GMII

As age (together with GMII) was significantly higher in the failed group (Table 2) and increasing age was shown to be a risk factor for postoperative failure on the univariate but not multivariate analysis (Table 3), we performed linear and logistic regression analyses for predicting the GMII value and category (low or high), respectively, using preoperative factors independent of GMII calculation, such as age, gender, CDR, previous phacoemulsification, DM, HT, and dyslipidemia, as possible 
Table 4 Logistic regression analysis of preoperative factors predicting failure of trabeculectomy at the 1-year follow-up using the Glaucoma Medication Intensity Index (GMII) $\geq 80$ cutoff to define "High GMII"

\begin{tabular}{|c|c|c|c|c|}
\hline \multirow[t]{2}{*}{ Variables } & \multicolumn{4}{|c|}{ Univariate analysis } \\
\hline & $\overline{\mathrm{OR}}$ & 95\% LB & $95 \%$ UB & $P$ value \\
\hline Age (years) & 1.07 & 1.01 & 1.15 & $0.04^{*}$ \\
\hline Gender (male) & 0.46 & 0.12 & 1.73 & 0.24 \\
\hline CDR & 0.97 & 0.93 & 1.03 & 0.30 \\
\hline Previous phacoemulsification & 3.33 & 0.93 & 12.66 & 0.07 \\
\hline Diabetes & 0.22 & 0.01 & 1.38 & 0.17 \\
\hline Systemic hypertension & 0.67 & 0.19 & 2.35 & 0.52 \\
\hline Dyslipidemia & 0.87 & 0.20 & 3.28 & 0.85 \\
\hline Medication exposure & 1.68 & 0.68 & 4.51 & 0.28 \\
\hline Medication requirement & 1.33 & 0.60 & 3.12 & 0.49 \\
\hline Treatment duration (years) & 1.12 & 0.93 & 1.36 & 0.23 \\
\hline IOP $(\mathrm{mmHg})$ & 1.04 & 0.96 & 1.13 & 0.34 \\
\hline High GMII & 9.75 & 1.66 & 187.00 & $0.04^{*}$ \\
\hline
\end{tabular}

confounding variables. Only increasing age was significantly correlated with both higher GMII (OR 10.42, 95\% CI 1.024-106.1; $P=0.05$ ) and classification as high GMII (OR 1.106, 95\% CI $1.03-1.21 ; P=0.01)$, while none of the other preoperative variables showed a significant association with GMII.

We also compared the IOP and glaucoma medications at baseline, and the IOP, glaucoma medications (and reduction from baseline), and number of bleb-needling procedures at the postoperative 1-year time point, between eyes with low and high GMII. These findings are summarized in Table 5. We did not compare the 2-year outcome of these variables due to the high number of dropouts at that time point. Both groups had similar IOP and number of glaucoma medications used at baseline. At the postoperative 1-year time point, IOP, number of glaucoma medications used, and number of bleb-needling procedures performed were significantly lower in the low GMII group (14.19 \pm $3.73 \mathrm{mmHg}, 0.44 \pm 1.03$, and $0.06 \pm 0.25$, respectively) than in the high GMII group $(17.45 \pm 5.32 \mathrm{mmHg}, \quad 1.88 \pm 1.92, \quad$ and
$0.48 \pm 1.03$, respectively; $P=0.03, P<0.01$, and $P=0.03$, respectively). The reduction in glaucoma medications at the 1-year follow-up from baseline was also significantly larger in eyes with low GMII $(2.81 \pm 1.47)$ compared to high GMII $(1.67 \pm 1.69)(P=0.02)$.

Finally, we attempted to explore the role of preservatives in glaucoma eye drops on postoperative outcome. However, as all study eyes $(n=55)$ were exposed to at least one of three preservatives preoperatively (benzalkonium chloride, polyquaternium-1, and stabilized oxychloro complex, it was not possible to make comparison with eyes not exposed to preservatives. Instead, we conducted regression analysis to assess the odds of failure with each preservative or combination of preservatives, but none were statistically significant, as shown in Table 6.

\section{DISCUSSION}

Multiple human and animal studies have shown that exposure to topical glaucoma 
Table 5 Postoperative mean intraocular pressure, number of bleb-needling procedures performed, and reduction of glaucoma medications (from baseline) at 1 year

\begin{tabular}{lllll}
\hline Variables & Overall & Low GMII $(<\mathbf{8 0})$ & High GMII $(\geq \mathbf{8 0})$ & $\boldsymbol{P}$ \\
\hline Number of eyes & 49 & 16 & 33 & - \\
Baseline IOP (mmHg) & $22.94 \pm 7.52$ & $21.75 \pm 6.67$ & $23.52 \pm 7.93$ & 0.45 \\
Baseline medications $(n)$ & $3.45 \pm 0.79$ & $3.25 \pm 0.93$ & $3.55 \pm 0.71$ & 0.22 \\
IOP at 1-year (mmHg) & $16.39 \pm 5.06$ & $14.19 \pm 3.73$ & $17.45 \pm 5.32$ & $0.03^{*}$ \\
Medications at 1-year $(n)$ & $1.41 \pm 1.80$ & $0.44 \pm 1.03$ & $1.88 \pm 1.92$ & $<0.01^{* *}$ \\
Medication reduction from baseline $(n)$ & $2.04 \pm 1.70$ & $2.81 \pm 1.47$ & $1.67 \pm 1.69$ & $0.02^{*}$ \\
Bleb-needling procedures $(n)$ & $0.35 \pm 0.88$ & $0.06 \pm 0.25$ & $0.48 \pm 1.03$ & $0.03^{*}$ \\
\hline
\end{tabular}

Values in table are presented as the mean $\pm S D$

Table 6 Logistic regression analysis of odds ratio for failure with different preservatives

\begin{tabular}{lllll}
\hline Preservative & OR & $\mathbf{9 5 \%}$ LB & 95\% UB & $\boldsymbol{P}$ \\
\hline BAK & 0.33 & 0.01 & 8.61 & 0.44 \\
PQ1 & 0.87 & 0.23 & 3.00 & 0.83 \\
SOC & 0.58 & 0.17 & 1.99 & 0.38 \\
BAK + PQ1 & 0.69 & 0.17 & 2.49 & 0.59 \\
BAK + SOC & 0.64 & 0.19 & 2.20 & 0.47 \\
PQ1 + SOC & 0.85 & 0.17 & 3.40 & 0.82 \\
BAK + PQ1 + SOC & 0.97 & 0.19 & 3.97 & 0.97 \\
\hline
\end{tabular}

$B A K$ Benzalkonium chloride, $O R$ odds ratio $P Q 1$ polyquaternium-1, SOC stabilized oxychloro complex

medications and their preservatives can induce subclinical chronic inflammation of the conjunctiva [11, 13, 15, 19-22], which predisposes to scarring of the filtration site following trabeculectomy and may limit postoperative success. This effect appears to be reflected in the multicenter Collaborative Initial Glaucoma Treatment Study (CIGTS), where using a mixed model-effects model the initial trabeculectomy group had lower predicted IOP at 5 years compared to the initial medication group, even when subsequent trabeculectomy was performed, suggesting a detrimental effect of glaucoma medication [23]. However, there have been few attempts at quantifying the association between preoperative use of topical glaucoma medication and postoperative outcome after trabeculectomy [12, 16]. These studies have reported better trabeculectomy outcome in eyes that used topical glaucoma medications for $<8$ weeks (compared to $>1$ year) [16], or $<3$ years (compared to $\geq 3$ years) with no difference between $<3$ months and $<3$ years [12]. We believe the highly variable (and sometimes contradictory) outcomes reported were likely due to the wide variance in the number of medications and/or dosing regimens used by subjects in these studies. Even when the number of medications was analyzed, it was often assumed, incorrectly, that the most recent (preoperative) prescriptions had remained unchanged since treatment initiation. Also, the generalizability of the results of these studies, conducted in the 1990s, to current practice is questionable, as the glaucoma medications commonly used at the time (such as miotics) have since changed dramatically, with an everincreasing number of newer drugs and fixedcombination eye drops becoming available [24]. This has generally allowed for a lower number of drops required daily, which should result in few ocular surface disorders and possibly better trabeculectomy outcome.

Based on the "intensity index" presented by Saade et al. from their work on the association between topical glaucoma medications and 
ocular surface disease [17], we further refined the parameter into a "Glaucoma Medication Intensity Index" (GMII) by additionally accounting for any variation in the treatment regimens during the entire treatment duration and weighing them proportionately in the index. Short of analyzing each individual medication and preservative used (which would be impractical in most real-world settings), our use of the GMII allows for a more accurate reflection of medication exposure than similar studies in the past.

Our study has shown that an increasing GMII value, which represents the combined accumulated dose of topical glaucoma medications and their accompanying preservatives, was associated with significantly higher odds for failure after trabeculectomy with MMC. Although age was noted to be significantly correlated with odds of failure on univariate analysis while GMII was not, this was reversed on subsequent multivariate analysis. The greater probability of older subjects to have a higher GMII in subsequent regression analyses for predicting GMII using factors independent of the GMII calculation can explain the higher mean age of our failed cases and also the higher risk of failure with increasing age on univariate analysis, but which are probably not intrinsically age-related, as shown with multivariate analysis in Table 3. It would appear that age was a confounding variable in our study, as older subjects were more likely to have glaucoma treatment initiated earlier, with longer duration of use and possibly a higher number of medications (added to the treatment over the years since diagnosis). This was confirmed when we examined factors not related to medication usage for predicting GMII, and age was shown to be significantly and positively correlated with the calculated value. The results suggest that the higher failure rate in our older subjects was not due to their age intrinsically, but because older subjects are more likely to have a higher GMII. Although we did not find age to be a significant risk factor for trabeculectomy failure, previous studies have arrived at conflicting conclusions, with age found not to be a risk factor in some studies $[25,26]$, and to be associated with lower [27] or higher [28] risk in others. In the study reporting higher risk for failure in subjects aged $>60$ years [28], treatment failure was only statistically significant for one success criteria (complete success) and not the other (qualified success), while treatment duration was not analyzed as a confounding variable in the authors' multivariate analysis despite the significantly longer duration of preoperative glaucoma medication use in the older-aged group (51.9 \pm 40 months) compared to the youngeraged group $(34.3 \pm 35.5$ months $)(P<0.006)$.

Our analyses further show that using a GMII $\geq 80$ to define high GMII was not only associated with a higher failure rate, but also with shorter survival time, higher mean postoperative IOP, greater number of needling procedures performed, and lower reduction of glaucoma medication requirement at the 1-year time point. In practical terms, this is equivalent to using eye drops once daily or twice daily for 11.5 or 6 years, respectively, or both these eye drops together for 4 years. Performing trabeculectomy before the GMII reaches 80 may, therefore, maximize the odds for a successful outcome. Note that the use of GMII $\geq 80$, based on further analysis as detailed in the Results section, should only be regarded as a useful reference for determining those eyes at high risk when planning glaucoma surgery. As our multivariate analysis showed, GMII as a surrogate marker of accumulated exposure to topical medications was a continuous variable directly correlated with the odds of failure (OR 1.021) so that the higher the GMII, the higher the risk of failure. This is analogous to the role of IOP in the risk of glaucoma development and progression, and similar to IOP, the GMII value should not be taken in isolation as there are likely other factors, such as use of preservativefree eye drops or presence of significant preoperative dry eye disease, influencing surgical success which were not investigated in this study.

There were several limitations to our study. Although our study was primarily focused on the accurate assessment of preoperative exposure to topical glaucoma medications, its retrospective nature made it difficult to accurately assess treatment compliance, as this may not always be reported by the patient or 
documented in the clinical notes. It is possible, or even probable, that subjects using $>2$ concurrent eye drops and/or who experienced any adverse drug effects may have poorer compliance and use less medications than the calculated GMII would indicate. This could mean that the GMII of some study eyes may be overestimated and the increased risk of trabeculectomy failure may occur at lower GMII level. In addition, the retrospective nature of our data means that documentation of preoperative glaucoma status (with perimetry or retinal nerve fiber layer analyses) was not standardized, although most subjects appear to have moderate to advanced disease, as reflected in the preoperative CDR, treatment duration, and medications used, and this was comparable between eyes with successful and failed outcomes. Our failure criteria for IOP level was chosen because it was more practical to use for a retrospective study examining the role of preoperative risk factors [25], and they are not meant to be used as guidelines for prospectively treating glaucoma patients, which should be based on the current severity of their disease with structural (optic disc morphology, peripapillary nerve fiber layer and ganglion cell-inner plexiform layer thickness) and functional (perimetry) assessments. It was also not possible for this study to compare the individual contribution of each glaucoma medication as all study eyes were using at least two medications (most were using 3-4), nor to analyze the contribution of different preservatives separate to the glaucoma medications, as all cases were exposed to some preservative in their eye drops; therefore, the GMII should be considered as a reflection of their combined effect. Our study was also limited by its relatively small sample size and large number of drop-outs after 1 year, which precluded meaningful subgroup (glaucoma type and medication) and longer-term analysis.

\section{CONCLUSIONS}

Our study has shown that higher accumulated preoperative exposure to topical glaucoma medications was associated with higher postoperative failure rate after primary trabeculectomy with MMC in POAG or NTG. Use of the GMII allows more accurate quantification of this preoperative exposure and helps the surgeon predict postoperative outcome. Higher GMII levels were correlated with greater odds of failure and eyes with a GMII $\geq 80$ had a significantly shorter survival time, higher mean IOP, greater needling rate, and less medication reduction. We hope our results will elicit interests in using the GMII to help predict high-risk cases that may benefit from additional perioperative intervention or treatment. It may also help with decision-making on the timing of glaucoma surgery before the GMII reaches a level associated with higher failure rate.

\section{ACKNOWLEDGEMENTS}

Perseus WF Wong and Jason CH Lam, both former research assistants at the Department of Ophthalmology, University of Hong Kong, are acknowledged for their assistance in the statistical analyses.

Funding. No funding or sponsorship was received for this study or publication of this article. The journal's Rapid Service Fee was funded by the authors.

Authorship. All named authors meet the International Committee of Medical Journal Editors (ICMJE) criteria for authorship for this article, take responsibility for the integrity of the work as a whole, and have given their approval for this version to be published.

Author Contributions. Jasper Ka Wai Wong: statistical analysis, drafting the manuscript, and reviewing final submission. Tsz Kin Leung: data collection, statistical analysis, and drafting the manuscript. Jimmy Shiu-ming Lai: drafting the manuscript and reviewing final submission. Jonathan Cheuk-hung Chan: concept and design, statistical analysis, drafting the manuscript, and reviewing final submission. 
Disclosures. None of the listed authors have any conflict of interest to declare with regard to the submitted article.

Compliance with Ethics Guidelines. Ethics approval for this study was obtained from the Institutional Review Board of the University of Hong Kong/Hospital Authority Hong Kong West cluster (reference number: UW 18-487). The study was conducted in accordance with the Declaration of Helsinki of 1964 and its later amendments.

Data Availability. The datasets generated during and/or analyzed during the current study are available from the corresponding author on reasonable request.

Open Access. This article is licensed under a Creative Commons Attribution-NonCommercial 4.0 International License, which permits any non-commercial use, sharing, adaptation, distribution and reproduction in any medium or format, as long as you give appropriate credit to the original author(s) and the source, provide a link to the Creative Commons licence, and indicate if changes were made. The images or other third party material in this article are included in the article's Creative Commons licence, unless indicated otherwise in a credit line to the material. If material is not included in the article's Creative Commons licence and your intended use is not permitted by statutory regulation or exceeds the permitted use, you will need to obtain permission directly from the copyright holder. To view a copy of this licence, visit http://creativecommons.org/licenses/by$\mathrm{nc} / 4.0 /$.

\section{REFERENCES}

1. Bourne RR, Taylor HR, Flaxman SR, et al. Number of people blind or visually impaired by glaucoma worldwide and in world regions 1990-2010: a metaanalysis. PLoS ONE. 2016;11(10):e0162229.

2. Tham YC, Li X, Wong TY, Quigley HA, Aung T, Cheng CY. Global prevalence of glaucoma and projections of glaucoma burden through 2040: a systematic review and meta-analysis. Ophthalmology. 2014;121(11):2081-90.

3. Heijl A, Leske MC, Bengtsson B, et al. Reduction of intraocular pressure and glaucoma progression: results from the Early Manifest Glaucoma Trial. Arch Ophthalmol. 2002;120(10):1268-79.

4. Quigley HA, Flower RW, Addicks EM, McLeod DS. The mechanism of optic nerve damage in experimental acute intraocular pressure elevation. Invest Ophthalmol Vis Sci. 1980;19(5):505-17.

5. Boland MV, Ervin AM, Friedman DS, et al. Comparative effectiveness of treatments for open-angle glaucoma: a systematic review for the U.S. Preventive services task force. Ann Intern Med. 2013;158(4):271-9.

6. Collaborative Normal-Tension Glaucoma Study Group. Comparison of glaucomatous progression between untreated patients with normal-tension glaucoma and patients with therapeutically reduced intraocular pressures. Am J Ophthalmol. 1998;126(4):487-97.

7. Kass MA, Heuer DK, Higginbotham EJ, et al. The Ocular Hypertension Treatment Study: a randomized trial determines that topical ocular hypotensive medication delays or prevents the onset of primary open-angle glaucoma. Arch Ophthalmol. 2002;120(6):701-13.

8. Gedde SJ, Herndon LW, Brandt JD, et al. Postoperative complications in the Tube Versus Trabeculectomy (TVT) study during five years of follow-up. Am J Ophthalmol. 2012;153(5):804-14.

9. Gedde SJ, Schiffman JC, Feuer WJ, et al. Treatment outcomes in the Tube Versus Trabeculectomy (TVT) study after five years of follow-up. Am J Ophthalmol. 2012;153(5):789-803.e2.

10. Conlon R, Saheb H, Ahmed II. Glaucoma treatment trends: a review. Can J Ophthalmol. 2017;52(1): 114-24.

11. Brandt JD, Wittpenn JR, Katz LJ, Steinmann WN, Spaeth GL. Conjunctival impression cytology in patients with glaucoma using long-term topical medication. Am J Ophthalmol. 1991;112(3): 297-301.

12. Broadway DC, Grierson I, O’Brien C, Hitchings RA. Adverse effects of topical antiglaucoma medication. II. The outcome of filtration surgery. Arch Ophthalmol. 1994;112(11):1446-54.

13. Noecker RJ, Herrygers LA, Anwaruddin R. Corneal and conjunctival changes caused by commonly used glaucoma medications. Cornea. 2004;23(5): 490-6. 
14. Ammar DA, Noecker RJ, Kahook MY. Effects of benzalkonium chloride-preserved, polyquad-preserved, and sofZia-preserved topical glaucoma medications on human ocular epithelial cells. Adv Ther. 2010;27(11):837-45.

15. Sherwood MB, Grierson I, Milgar L, Hitchings RA. Long-term morphologic effects of antiglaucoma drugs on the conjunctiva and Tenon's capsule in glaucomatous patients. Ophthalmology. 1989;96(3):327-35.

16. Lavin MJ, Wormald RL, Migdal CS, Hitchings RA. The influence of prior therapy on the success of trabeculectomy. Arch Ophthalmol. 1990;108(11): 1543-8.

17. Saade CE, Lari HB, Berezina TL, Fechtner RD, Khouri AS. Topical glaucoma therapy and ocular surface disease: a prospective, controlled cohort study. Can J Ophthalmol. 2015;50(2):132-6.

18. Bursac Z, Gauss CH, Williams DK, Hosmer DW. Purposeful selection of variables in logistic regression. Source Code Biol Med. 2008;3:17.

19. Broadway DC, Grierson I, O’Brien C, Hitchings RA. Adverse effects of topical antiglaucoma medication. I. The conjunctival cell profile. Arch Ophthalmol. 1994;112(11):1437-45.

20. Mietz H, Niesen U, Krieglstein GK. The effect of preservatives and antiglaucomatous medication on the histopathology of the conjunctiva. Graefes Arch Clin Exp Ophthalmol. 1994;232(9):561-5.

21. Baudouin C, Pisella PJ, Fillacier K, et al. Ocular surface inflammatory changes induced by topical antiglaucoma drugs: human and animal studies. Ophthalmology. 1999;106(3):556-63.

22. Baudouin C, Hamard P, Liang H, Creuzot-Garcher C, Bensoussan L, Brignole F. Conjunctival epithelial cell expression of interleukins and inflammatory markers in glaucoma patients treated over the long term. Ophthalmology. 2004;111(12):2186-92.

23. Musch DC, Gillespie BW, Niziol LM, Cashwell LF, Lichter PR, Collaborative Initial Glaucoma Treatment Study G. Factors associated with intraocular pressure before and during 9 years of treatment in the collaborative initial glaucoma treatment study. Ophthalmology. 2008;115(6):927-33.

24. Soltau JB, Zimmerman TJ. Changing paradigms in the medical treatment of glaucoma. Surv Ophthalmol. 2002;47(Suppl 1):S2-5.

25. Duman F, Waisbourd M, Faria B, Addis V, Hsieh M, Ekici F, et al. Trabeculectomy in patients with glaucoma over 80 years of age: relatively short-term outcomes. J Glaucoma. 2016;25(3):e123-7.

26. Briggs MC, Jay JL. Age over 46 years does not affect the pressure lowering effect of trabeculectomy in primary open angle glaucoma. Br J Ophthalmol. 1999;83(3):280-4.

27. The AGIS Investigators. The Advanced Glaucoma Intervention Study (AGIS): 11. Risk factors for failure of trabeculectomy and argon laser trabeculoplasty. Am J Ophthalmol. 2002;134(4):481-98.

28. Hoang TKH, Kim YK, Jeoung JW, Park KH. Relationship between age and surgical success after trabeculectomy with adjunctive mitomycin C. Eye (Lond). 2018;32(8):1321-8. 